\title{
Advances in coronary molecular imaging: Leveraging the power of image processing
}

\author{
Amr Abdelbaky, MD, ${ }^{a}$ Georges El Fakhri, PhD, ${ }^{b}$ and Ahmed Tawakol, $M D^{a, c}$ \\ a Cardiac MR PET CT Program, Massachusetts General Hospital, Harvard Medical School, \\ Boston, MA \\ b Gordon Center for Medical Imaging, Department of Radiology, Massachusetts General \\ Hospital, Harvard Medical School, Boston, MA \\ c Nuclear Cardiology, Division of Cardiology, Department of Medicine, Massachusetts General \\ Hospital, Harvard Medical School, Boston, MA
}

Received Aug 8, 2018; accepted Aug 9, 2018

doi: $10.1007 / \mathrm{s} 12350-018-1454-\mathrm{x}$

\section{See related article, pp. 494-504}

Coronary vascular events are most often caused by rupture of atherosclerotic plaques. Prior to their rupture, such plaques are likely to have at least one of several high-risk structural or biological processes known to associate with increased risk of atherothrombosis. Thus, efforts have long been directed to identify these highrisk features non-invasively. While current imaging modalities are adept at measuring high-risk structural features, such as luminal stenosis and vessel wall morphology, they cannot directly report on the important high-risk biological features. On the other hand, molecular imaging techniques, such as positron emission tomography (PET) coupled with sensitive probes provide a unique opportunity to assess atherosclerotic plaque biology, and have the potential to complement structural information and thus, improve risk stratification and enable enhanced monitoring of therapeutic interventions.

Among biological processes that increase atherothrombosis risk, two stand out prominently: atherosclerotic inflammation and plaque microcalcification. ${ }^{1}$ The important role for inflammation in atherosclerosis is well established. Atherosclerosis is a

Reprint requests: Ahmed Tawakol, MD, Cardiac MR PET CT Program, Massachusetts General Hospital, Harvard Medical School, Boston, MA; atawakol@mgh.harvard.edu

J Nucl Cardiol 2020;27:505-7.

$1071-3581 / \$ 34.00$

Copyright (C) 2018 American Society of Nuclear Cardiology. chronic inflammatory condition, where inflammation participates in all phases of atherosclerotic disease, from its initiation to progression, to atherothrombosis. ${ }^{1}$ Recently, the CANTOS trial provided key evidence for a causal role for inflammation, by demonstrating that selective anti-inflammatory therapy decreases the risk of cardiovascular disease (CVD) events. ${ }^{2}$ Likewise, the process of microcalcification can also potentiate CVD risk. Microcalcification (not to be conflated with macrocalcification) often occurs near the fibrous cap, and may contribute to plaque destabilization by increasing mechanical stress. Hence localization of active inflammation or microcalcification has the potential to identify plaques that are prone to rupture.

Several PET radiotracers have been employed to measure these biological processes within atheroma. ${ }^{1}$ Among these, 2 radiotracers are widely available: ${ }^{18} \mathrm{~F}$ fluorodeoxyglucose $\left({ }^{18} \mathrm{~F}-\mathrm{FDG}\right)$, a marker of vascular metabolic activity and inflammation, and ${ }^{18} \mathrm{~F}$-sodium fluoride $\left({ }^{18} \mathrm{~F}-\mathrm{NaF}\right)$, a marker of active microcalcification. ${ }^{18}$ F-FDG uptake accumulates within inflammatory cells that reside in the atheroma. ${ }^{3}$ Measurement of FDG uptake within the artery wall provides a non-invasive index of atherosclerotic inflammation. ${ }^{3,4}{ }^{18} \mathrm{~F}-\mathrm{NaF}$ binds to hydroxyl groups in hydroxyapatite, and is used clinically to detect tumors that have metastasized to bone. It's binding to hydroxyapatite is also leveraged to detect the process of plaque microcalcification. ${ }^{5}$

PET imaging of plaque biology can yield powerful insights. Quantification of arterial inflammation, using 18FDG PET imaging, provides incremental information for predicting the risk of incident cardiovascular disease events $^{6}$ and can be used to track treatment-related changes in atherosclerotic inflammation. ${ }^{7}$ Likewise, imaging atherosclerotic microcalcification with $18 \mathrm{~F}$ - 
$\mathrm{NaF}$ has also produced important insights. $18 \mathrm{~F}-\mathrm{NaF}$ uptake is increased in recent culprit lesions involved in atherothrombosis. ${ }^{8} 9$ Recently, $18 \mathrm{NaF}$ uptake within abdominal aortic aneurysms was found to predict aneurysm growth and future clinical events. ${ }^{10}$

However, the majority of atherosclerotic plaque imaging has thus far focused on larger vessels, such as the aorta and carotids, given the greater ease of imaging large vessels, and the relative availability of histopathological samples for validation studies (such as from endarterectomy specimens). Coronary imaging poses significant technical challenges. Firstly, coronary vessels have a small caliber relative to PET spatial resolution (4-6 mm), subjecting them to substantial partial volume effects that ultimately underestimate vessel wall activity. Secondly, epicardial coronary vessels (particularly right coronary artery and distal coronary tree) are subject to significant motion due to respiration and myocardial contractility. This can lead to significant blurring of PET signal, further underestimation of wall activity and undermining of accuracy and reproducibility of the derived measurements. None-theless, several groups have demonstrated feasibility of coronary imaging using PET tracers, though with varying degrees of success. While coronary imaging using 18FDG has been shown to be feasible, ${ }^{11,12}$ competing FDG uptake from the adjacent myocardium substantially limits its practical use. ${ }^{18} \mathrm{~F}-\mathrm{NaF}$, on the other hand, is more likely than FDG to identify coronary plaques, in part owing to the fact that there is less competing uptake from the adjacent myocardium. ${ }^{9}$ While these efforts demonstrate initial feasibility of coronary imaging, widespread clinical adoption of these approaches is still not possible given the relatively low signal to noise ratio, and the lack of a standardized approach for image reconstruction and analysis. Hence, novel techniques are needed in order to reduce noise and improve the ability to accurately quantify coronary plaque biological activity.

The study by Doris et al., published in this issue of the Journal of Nuclear cardiology, achieves some of these goals. The authors sought to investigate the impact of reconstruction parameters and cardiac motion-correction on $18 \mathrm{~F}$ Sodium Fluoride $(18 \mathrm{~F}-\mathrm{NaF})$ PET imaging of coronary plaques in humans. This work extended the findings of prior work by the same group, demonstrating that a novel cardiac motion-correction method, improves PET quantification. ${ }^{13}$ The current study further optimized that approach. They conducted $18 \mathrm{NaF}$ PET imaging in 22 individuals who experienced a recent (within 22 days) acute coronary syndrome, and who had undergone invasive coronary angiography where a culprit lesion was identified. Using the acquired PET datasets, the authors initially tested different reconstruction algorithms in a subset of subjects, and identified the algorithm with the best image quality and signal to noise ratio (SNR). Then, across all subjects they compared the novel motion-correction algorithm to: (A) un-corrected images and (B) images derived from the diastolic phase. The authors observed that the novel approach improved overall image quality and SNR, and yielded better discrimination between culprit and non-culprit plaques. Based on these findings, the authors recommended using the novel reconstruction and motion-correction techniques in future coronary ${ }^{18} \mathrm{~F}-\mathrm{NaF}$ PET studies.

This study highlights several important points. Firstly, innovations in image processing can importantly enhance the utility of coronary plaque imaging with PET molecular imaging techniques. Secondly, as the authors suggested, these efforts should be further optimized, to additionally take into account partial volume effects. The latter is no easy task given the potential for introducing spurious bias associated with inaccurate modeling of the detector point spread function. Such future studies should evaluate the additive impact of partial volume correction on image quality and quantification accuracy. ${ }^{14}$ Moreover, these approaches should be validated in prospective outcome studies. Should such studies continue to demonstrate that these reconstruction approaches enhance the accuracy of coronary plaque imaging, they may facilitate the emergence of PET coronary imaging as an important tool. Finally, in order for the proposed approaches to be widely adopted in the clinical setting, massive computing acceleration should take place. The use of GPU computing is a step in the right direction.

Indeed, new non-invasive tools are needed to better target therapies in patients with atherosclerotic disease. Despite advances in therapy and diagnosis, atherosclerosis remains the leading cause of death in the western world. Novel therapies (e.g., PCSK9 inhibitors and Canakinumab) have recently been shown to significantly decrease cardiovascular disease events. While promising, these novel therapies are highly expensive and carry additional risks, thus highlighting the importance of better tools to assess cardiovascular disease risk. In the emerging era of personalized medicine and rising healthcare costs, molecular imaging has the potential to further refine individual risk and to better-direct such expensive or risky therapies to those patients that would derive the greatest benefit. Furthermore, these imaging techniques could be leveraged to identify novel therapies directed against atherosclerotic disease, much in the way that novel drugs have been studied in the large arteries. ${ }^{7}$ These reconstruction techniques may facilitate the development of emerging tracers, and could accelerate the development of more effective PET tracers. 
These inter-related efforts may play important roles in reducing the burden of atherosclerotic disease events.

To further rise to the challenge, parallel efforts will be needed to in order enhance the diagnostic accuracy of coronary molecular imaging techniques. This should be achieved on multiple fronts, through: advancing PET imaging system development (i.e., innovations in PET/ $\mathrm{CT}$ and PET/MR); developing novel radiotracers; and refining reconstruction and motion-correction techniques. The study by Doris et al. is an important step toward this goal.

\section{Disclosure}

The authors have no disclosures relevant to the subject matter.

\section{References}

1. Teague HL, Ahlman MA, Alavi A, et al. Unraveling vascular inflammation: From immunology to imaging. J Am Coll Cardiol. 2017;70:1403-12. https://doi.org/10.1016/j.jacc.2017.07.750.

2. Ridker PM, Everett BM, Thuren T, et al. Antiinflammatory therapy with canakinumab for atherosclerotic disease. N Engl J Med. 2017;377:1119-31. https://doi.org/10.1056/nejmoa1707914.

3. Tawakol A, Migrino RQ, Bashian GG, et al. In vivo 18F-fluorodeoxyglucose positron emission tomography imaging provides a noninvasive measure of carotid plaque inflammation in patients. $\mathrm{J}$ Am Coll Cardiol. 2006;48:1818-24. https://doi.org/10.1016/j.jacc. 2006.05.076.

4. Rudd JH, Warburton EA, Fryer TD, et al. Imaging atherosclerotic plaque inflammation with [18F]-fluorodeoxyglucose positron emission tomography. Circulation. 2002;105:2708-11.

5. Irkle A, Vesey AT, Lewis DY, et al. Identifying active vascular microcalcification by $18 \mathrm{~F}$-sodium fluoride positron emission tomography. Nat Commun. 2015. https://doi.org/10.1038/nc omms 8495 .

6. Figueroa AL, Abdelbaky A, Truong QA, et al. Measurement of arterial activity on routine FDG PET/CT images improves prediction of risk of future CV events. JACC Cardiovasc Imaging. 2013;6:1250-9. https://doi.org/10.1016/j.jcmg.2013.08.006.

7. Tawakol A, Fayad ZA, Mogg R, et al. Intensification of statin therapy results in a rapid reduction in atherosclerotic inflammation: Results of a multicenter fluorodeoxyglucose-positron emission tomography/computed tomography feasibility study. J Am Coll Cardiol. 2013;62:909-17.

8. Vesey AT, Jenkins WSA, Irkle Agnese, et al. 18F-fluoride and 18Ffluorodeoxyglucose positron emission tomography after transient ischemic attack or minor ischemic strokeCLINICAL PERSPECTIVE: Case-control study. Circulation. 2017;10:e004976. https://d oi.org/10.1161/circimaging.116.004976.

9. Joshi NV, Vesey AT, Williams MC, et al. 18F-fluoride positron emission tomography for identification of ruptured and high-risk coronary atherosclerotic plaques: A prospective clinical trial. Lancet. 2014;383:705-13. https://doi.org/10.1016/s0140-6736(13 61754-7.

10. Forsythe RO, Dweck MR, McBride OMB, et al. 18F-sodium fluoride uptake in abdominal aortic aneurysms: The SoFIA3 study. J Am Coll Cardiol. 2018;71:513-23. https://doi.org/10.1016/j.jacc. 2017.11.053.

11. Rogers IS, Tawakol A. Imaging of coronary inflammation with FDG-PET: Feasibility and clinical hurdles. Curr Cardiol Rep. 2011;13:138-44. https://doi.org/10.1007/s11886-011-0168-3.

12. Cheng VY, Slomka PJ, Meunier LL, et al. Coronary arterial 18FFDG uptake by fusion of PET and coronary CT angiography at sites of percutaneous stenting for acute myocardial infarction and stable coronary artery disease. J Nucl Med. 2012;53:575-83. https://doi.org/10.2967/jnumed.111.097550.

13. Rubeaux M, Joshi NV, Dweck MR, et al. Motion correction of $18 \mathrm{~F}-\mathrm{NaF}$ PET for imaging coronary atherosclerotic plaques. $\mathrm{J}$ Nucl Med. 2016;57:54-9.

14. Huet P, Burg S, Guludec DL, et al. Variability and uncertainty of 18F-FDG PET imaging protocols for assessing inflammation in atherosclerosis: Suggestions for improvement. J Nucl Med. 2015;56:552-9. https://doi.org/10.2967/jnumed.114.142596. 